\title{
Resveratrol-induced autophagy and apoptosis in cisplatin-resistant human oral cancer CAR cells: A key role of AMPK and Akt/mTOR signaling
}

\author{
CHAO-HSIANG CHANG ${ }^{1}$, CHAO-YING LEE ${ }^{2}$, CHI-CHENG LU ${ }^{3}$, FUU-JEN TSAI ${ }^{4,5}$, YUAN-MAN HSU ${ }^{6}$, \\ JE-WEI TSAO $^{3}$, YU-NING JUAN ${ }^{3}$, HONG-YI CHIU $^{7}$, JAI-SING YANG $^{3}$ and CHING-CHIUNG WANG ${ }^{1}$ \\ ${ }^{1}$ School of Pharmacy, College of Pharmacy, Taipei Medical University, Taipei 110; ${ }^{2}$ School of Pharmacy, \\ China Medical University; ${ }^{3}$ Department of Medical Research, China Medical University Hospital, China Medical \\ University; ${ }^{4}$ Human Genetic Center, China Medical University Hospital; ${ }^{5}$ School of Post-Baccalaureate Chinese \\ Medicine, China Medical University; ${ }^{6}$ Department of Biological Science and Technology, China Medical University, \\ Taichung 404; ${ }^{7}$ Department of Pharmacy, Buddhist Tzu Chi General Hospital, Hualien 970, Taiwan, R.O.C.
}

Received December 1, 2016; Accepted January 23, 2017

DOI: $10.3892 /$ ijo.2017.3866

\begin{abstract}
Resveratrol is known to be an effective chemopreventive phytochemical against multiple tumor cells. However, the increasing drug resistance avoids the cancer treatment in oral cavity cancer. In this study, we investigated the oral antitumor activity of resveratrol and its mechanism in cisplatin-resistant human oral cancer CAR cells. Our results demonstrated that resveratrol had an extremely low toxicity in normal oral cells and provoked autophagic cell death to form acidic vesicular organelles (AVOs) and autophagic vacuoles in CAR cells by acridine orange (AO) and monodansylcadaverine (MDC) staining. Either DNA fragmentation or DNA condensation occurred in resveratrol-triggered CAR cell apoptosis. These inhibitors of PI3K class III (3-MA) and AMP-activated protein kinase (AMPK) (compound c) suppressed the autophagic vesicle formation, LC3-II protein levels and autophagy induced by resveratrol. The pan-caspase inhibitor Z-VAD-FMK attenuated resveratrol-triggered cleaved caspase-9, cleaved caspase-3 and cell apoptosis. Resveratrol also enhanced phosphorylation of AMPK and regulated autophagy- and pro-apoptosis-related signals in resveratrol-treated CAR cells. Importantly, resveratrol also stimulated the autophagic mRNA gene expression, including Atg5, Atg12, Beclin-1 and LC3-II in CAR cells.
\end{abstract}

Correspondence to: Dr Ching-Chiung Wang, School of Pharmacy, College of Pharmacy, Taipei Medical University, $250 \mathrm{Wu}-\mathrm{Hsing}$ Street, Taipei City 11042, Taiwan, R.O.C.

E-mail: crystal@tmu.edu.tw

Dr Jai-Sing Yang, Department of Medical Research, China Medical University Hospital, China Medical University, 2 Yuh-Der Road, Taichung City 40447, Taiwan, R.O.C.

E-mail: jaisingyang@gmail.com

Key words: resveratrol, autophagic death, apoptosis, AMP-activated protein kinase, cisplatin-resistant oral cancer cell
Overall, our findings indicate that resveratrol is likely to induce autophagic and apoptotic death in drug-resistant oral cancer cells and might become a new approach for oral cancer treatment in the near future.

\section{Introduction}

Apoptosis and autophagy are two main modes of programmed cell death (1). Apoptosis is regulated by caspases and Bcl-2 family proteins and involved in multiple cellular signaling pathways (2). Autophagy is modulated as a survival mechanism induced in problematic environment to maintain cell integrity by nutrient starvation and/or metabolic stress (3). Recently, apoptosis and autophagy may be triggered by general upstream signaling to affect tumor cell development and therapy $(4,5)$.

Apoptosis is characterized by cellular morphological membrane blebbing, cell shrinkage, nuclear fragmentation, chromatin and DNA condensation, and chromosomal DNA fragmentation $(1,6)$. There are two major mechanisms involved in apoptotic cell death such as intrinsic (mitochondria-mediated) and extrinsic (death receptor) pathways (7-9). In the intrinsic pathway, the first regulatory stage is mediated by Bcl-2 family proteins. In apoptotic stimuli, the level of Bax (a pro-apoptotic protein) increased, following binding to Bcl-2 (a pro-survival protein) then releasing Bax/Bak molecules. Free Bax and Bak form oligomers leading to cytochrome $c$ release from mitochondria to the cytoplasm. The released cytochrome $c$ activates the caspase-9/-3 cascade to induce apoptosis. The second regulatory step of mitochondrial pathway is the formation of apoptosome. The mitochondria-related proteins (such as cytochrome $c$, Apaf-1 and pro-caspase-9) can be released to the cytoplasm to form apoptosome, which binds and cleaves initiator pro-caspase- 9 and activates the caspase-9/-3 cascade to induce apoptosis $(7,10)$.

Autophagy is an important response to the cellular environments and positively regulates cellular processes for survival or death during cell stress and damage, nutrient starvation, aging and pathogen infection $(11,12)$. Morphological and biochemical 
modification involve autophagy-related proteins (Atgs) and related autophagy signal molecules (13). Once autophagic response is activated, the membrane nucleation is regulated by the signal cascade of phosphatidylinositol 3-kinase (PI3K) class III, Beclin-1, Rubicon and Atg14. Furthermore, Atg16L1Atg12-Atg7-Atg5 complexes and microtubule-associated protein 1 light chain 3 type II (LC3-II) are required for autophagosome formation before the cytoplasm and phagophore of various organelles are sequestered $(13,14)$. Autophagolysosome is from the autophagosome fusion with the lysosome to induce the degradation of the captured proteins or organelles. The autophagosomal marker LC3-II increases from the conversion of LC3-I during undergoing autophagic death $(12,13)$. Thus, autophagy is developing as a new target for cancer therapy and chemoprevention (15), and induction of autophagic cell death is considered to possibly be one of the best strategies in cancer chemotherapy.

Resveratrol (3,4',5-trihydroxy-trans-stilbene) is a polyphenolic compound and abundantly found in plants and foods (16-19). A wide spectrum of pharmacological bio-activities (antioxidant, anti-inflammatory, anti-atherosclerotic and antitumor properties) of resveratrol have been reported $(16,20)$. Resveratrol was first found as a potential antitumor agent (21). A variety of biological effects of resveratrol include anti-proliferation, inhibition of angiogenesis and anti-metastatic activities, as well as inhibition of tumorigenesis $(22,23)$. Although the antitumor effects of resveratrol have been found to modulate various steps of carcinogenesis and development, its underlying mechanism of anticancer effect regarding autophagy and apoptosis is unclear in resistant human oral cancer cells. The purpose of this study was to develop an understanding of the effects of resveratrol on cisplatin-resistant human oral cancer CAR cells and to further determine its therapeutic value and anticancer activity in treating oral cancer cells in vitro.

\section{Materials and methods}

Chemicals and reagents. $\beta$-actin antibody, cisplatin, 3-methyladenine (3-MA), monodansylcadaverine (MDC), and thiazolyl blue tetrazolium bromide (MTT) were obtained from Sigma-Aldrich (St. Louis, MO, USA). Acridine orange (AO), LysoTracker Red DND-99, and trypsin-EDTA were purchased from Thermo Fisher Scientific (Waltham, MA, USA). The primary antibodies against cytochrome $c$, Apaf-1, AIF, Endo G, Bcl-2, and Bax and the goat anti-rabbit or anti-mouse IgG-horseradish peroxidase (HRP) secondary antibodies were purchased from Santa Cruz Biotechnology (Santa Cruz, CA, USA). The other primary antibodies used in this study were from Cell Signaling Technology (Danvers, MA, USA). Compound $\mathrm{c}$ and Z-VAD-FMK were from Merck Millipore (Billerica, MA, USA).

Cell culture. CAR, a cisplatin-resistant cell line, was developed by exposing parental human tongue squamous cell carcinoma CAL 27 cell line (American Type Culture Collection, Manassas, VA, USA) to $10-80 \mu \mathrm{M}$ of cisplatin, and CAR cells stably resistant to cisplatin as previously described $(24,25)$. CAR cells were cultured in Dulbecco's modified Eagle's medium (DMEM) supplemented with $10 \%$ fetal bovine serum (FBS), $100 \mathrm{U} / \mathrm{ml}$ penicillin, $100 \mu \mathrm{g} / \mathrm{ml}$ streptomycin, and
Table I. Primers for quantitative RT-PCR analysis.

\begin{tabular}{ll}
\hline Primer name & \multicolumn{1}{c}{ Nucleotide sequence (5' to 3') } \\
\hline Atg5 & F: TTTCCTCCACTGCCATCATTAA \\
& R: GGCCAAAGGTTTCAGCTTCA \\
Atg12 & F: TGTGGCCTCAGAACAGTTGTTTA \\
& R: CGCCTGAGACTTGCAGTAATGT \\
Beclin-1 & F: GGATGGTGTCTCTCGCAGATTC \\
& R: GGTGCCGCCATCAGATG \\
LC3-II & F: CCGACCGCTGTAAGGAGGTA \\
& R: AGGACGGGCAGCTGCTT \\
GAPDH & F: ACACCCACTCCTCCACCTTT \\
& R: TAGCCAAATTCGTTGTCATACC \\
\hline
\end{tabular}

F, forward; R, reverse.

$2 \mathrm{mM}$ L-glutamine (Thermo Fisher Scientific) in an incubator at $37^{\circ} \mathrm{C}$ with a humidified $5 \% \mathrm{CO}_{2}$. Cisplatin-resistant CAR cells were continuously cultured in $80 \mu \mathrm{M}$ cisplatin, unless otherwise indicated. Human normal gingival fibroblast (HGF) cells were a kind gift of Dr Tzong-Ming Shieh (Department of Dental Hygiene, China Medical University) and cultured in DMEM with $10 \%$ FBS, $100 \mathrm{U} / \mathrm{ml}$ penicillin and $100 \mu \mathrm{g} / \mathrm{ml}$ streptomycin as previously described (26).

Cell viability assay. CAR or HGF cells ( $1 \times 10^{4}$ cells/well) were plated in 96-well plates in $100 \mu 1$ medium with or without 25 , $50,75,100$ and $200 \mu \mathrm{M}$ of resveratrol for $48 \mathrm{~h}$. CAR cells were individually pretreated with $10 \mathrm{mM} 3-\mathrm{MA}$ (a PI3K class III inhibitor), $10 \mu \mathrm{M}$ compound c (an AMPK inhibitor) and $15 \mu \mathrm{M}$ Z-VAD-FMK (a pan-caspase inhibitor) for $1 \mathrm{~h}$, followed by treatment with or without $50 \mu \mathrm{M}$ resveratrol for $48 \mathrm{~h}$. After that, DMEM containing $500 \mu \mathrm{g} / \mathrm{ml}$ MTT was added, and medium was then aspirated from each well to detect viability as described elsewhere $(27,28)$. Cell morphological examination of apoptotic characteristics and autophagic vacuoles was determined and photographed utilizing a phase-contrast microscope as previously described (29).

Observations for autophagic vacuoles and autophagy marker. CAR cells $\left(5 \times 10^{4}\right.$ cells $\left./ \mathrm{ml}\right)$ were plated on sterile coverslips in tissue culture plates and treated with $50 \mu \mathrm{M}$ resveratrol for $48 \mathrm{~h}$. Cells were then individually stained with either $1 \mu \mathrm{g} / \mathrm{ml}$ AO, $1 \mu \mathrm{g} / \mathrm{ml}$ LysoTracker Red DND-99 or $100 \mu \mathrm{M}$ MDC for $10 \mathrm{~min}$ as previously described $(13,30)$. The autophagy marker LC3 was assayed using the Premo Autophagy Sensor LC3B-GFP (BacMam 2.0) kit (Thermo Fisher Scientific) as specified by the manufacturer. The occurrence of autophagic vacuoles, acidic vesicular organelles (AVOs) and LC3B-GFP were immediately monitored by fluorescence microscopy (Nikon, Melville, NY, USA).

TUNEL/DAPI staining. CAR cells $\left(2 \times 10^{5}\right.$ cells/well) into 12 -well plates were incubated with $50 \mu \mathrm{M}$ resveratrol. After exposure for $48 \mathrm{~h}$, apoptotic DNA breaks were detected utilizing 
$\mathbf{A}$

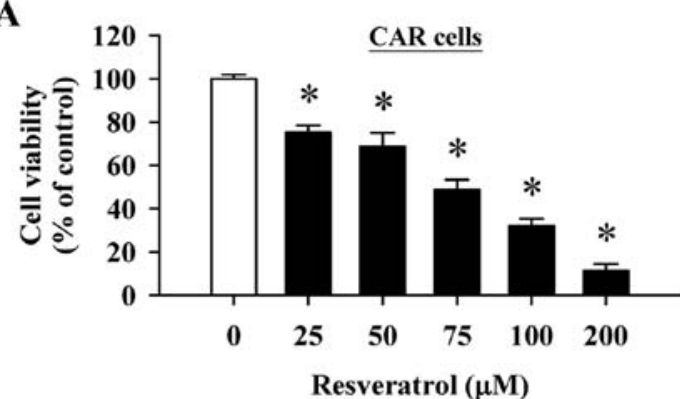

B

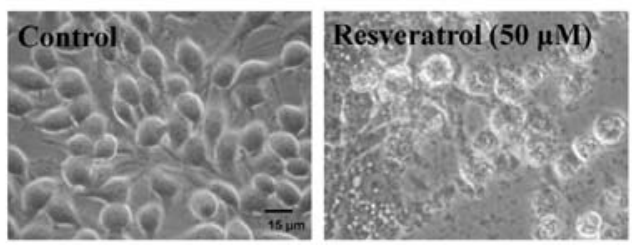

C

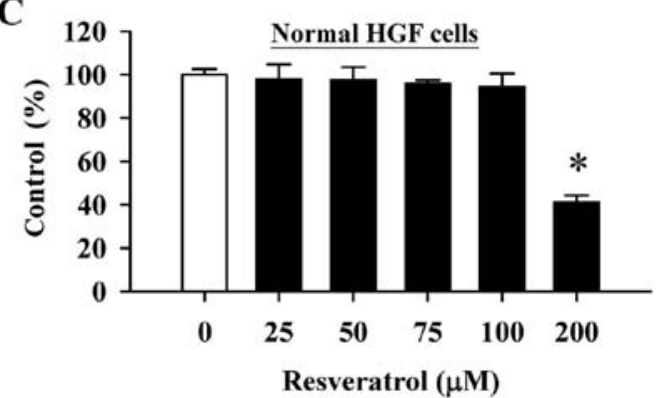

D
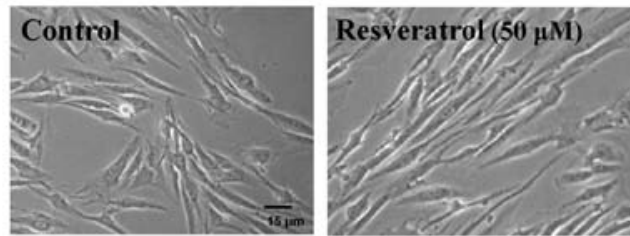

Figure 1. Effect of resveratrol on CAR and normal oral cell viability and morphological examination. CAR and normal human gingival fibroblast (HGF) cells were treated with or without indicated concentrations of resveratrol for $48 \mathrm{~h}$. Cell viability was individually measured by an MTT assay in (A) CAR and (C) normal HGF cells. The data are plotted as the mean \pm SEM $(n=3)$. ${ }^{*}<<0.05$ compared to untreated control. The effects of morphological changes on (B) CAR and (D) normal HGF cells were photographed with a phase-contrast microscope. Scale bar, $15 \mu \mathrm{m}$.

the In Situ Cell Death Detection kit, Fluorescein (Roche Diagnostics GmbH, Roche Applied Science, Mannheim, Germany) following the protocol provided by the manufacturer. Cells were counter-stained with $1 \mu \mathrm{g} / \mathrm{ml}$ 4',6-diamidino-2-phenylindole (DAPI, Thermo Fisher Scientific) and photographed using a fluorescence microscope.

Western blotting. CAR cells $\left(5 \times 10^{6}\right.$ cells/T-75 flask) were exposed to resveratrol $(0,25,50$ and $100 \mu \mathrm{M})$ for indicated intervals of time. Whole-cell lysates were isolated, and quantification of the protein concentration was completed by Bradford assay (Bio-Rad Protein Assay, Bio-Rad Laboratories, Hercules, CA, USA) as previously described (31). Equal amounts of protein samples after boiling and the addition of denaturing sample buffer were separated using a $10 \%$ or $12 \%$ SDS-PAGE and further employed by immunoblotting as detailed by Chiang et al (29). All bands were normalized to the level of $\beta$-actin for each lane.

Quantitative RT-PCR. CAR cells ( $5 \times 10^{6}$ cells per T-75 flask) were treated with or without $50 \mu \mathrm{M}$ resveratrol for $24 \mathrm{~h}$. Cells were collected, and cell pellets were collected by centrifugation to extract total RNA by the Qiagen RNeasy Mini kit (Qiagen, Valencia, CA, USA). RNA purity was detected, and RNA sample was individually reverse-transcribed using the High Capacity cDNA Reverse Transcription kits (Applied Biosystems/Thermo Fisher Scientific). Quantitative PCR was assessed to amplify with 2X SYBR Green PCR Master Mix (Applied Biosystems/ Thermo Fisher Scientific), and forward and reverse primers as listed in Table I were determined as previously reported $(13,32)$. Applied Biosystems 7300 Real-time PCR System was employed at least in triplicate, and each value was performed in the comparative threshold cycles $\left(\mathrm{C}_{\mathrm{T}}\right)$ method to normalize to the housekeeping gene GAPDH.

Statistical analysis. All data are reported as the mean \pm SEM of triplicate samples. The significant differences of data were subjected to Student's t-test for comparison of two groups as described in figure legends, and P-value $<0.05$ was considered to indicate a statistically significant difference.

\section{Results}

Resveratrol exhibits cytotoxicity and suppresses CAR cell viability. Resveratrol concentration-dependently reduced viable CAR cells (Fig. 1A). The 50\% maximal inhibitory concentration $\left(\mathrm{IC}_{50}\right)$ of resveratrol for a $24-, 48$ - and $72-\mathrm{h}$ treatments in CAR cells were 95.23 $\pm 3.26,73.23 \pm 2.29$ and $51.62 \pm 3.36 \mu \mathrm{M}$, respectively. Resveratrol was also found to induce the formation of autophagic vacuoles and apoptotic bodies in CAR cells after $50 \mu \mathrm{M}$ resveratrol challenge for $48 \mathrm{~h}$ (Fig. 1B). This finding implies that autophagic cell death and apoptotic effects play pivotal roles in resveratrol-treated CAR cells. Importantly, no cytotoxic effect or morphological trait change on resveratrol-treated normal HGF cells were observed (Fig. 1C and D), suggesting that resveratrol has low toxicity in normal oral cells. The $\mathrm{IC}_{50}$ value of HGF cells is over $100 \mu \mathrm{M}$ resveratrol. Therefore, resveratrol reduced viability in CAR cells instead of normal HGF cells possibly through autophagic and apoptotic mechanisms.

Resveratrol elicits autophagic and apoptotic death in CAR cells. To further test the autophagic cell death caused by resveratrol, the formation of autophagosome vesicles in CAR cells was monitored. Resveratrol triggered the clear occurrence of AVOs within the cytoplasm in comparison to control using AO staining (Fig. 2A). The autophagic evidence was also found after resveratrol-treated CAR cells were individually stained with LysoTracker Red DND-99 and MDC, which are widely used fluorescent probes to preferentially accumulate in autophagic vacuoles. After $50 \mu \mathrm{M}$ resveratrol exposure for $48 \mathrm{~h}$, autophagic vacuoles were observed, and the fluorescent intensity of MDC staining was directly proportional to $50 \mu \mathrm{M}$ resveratrol (Fig. 2A). The LC3 distribution of resveratrol-treated 
A

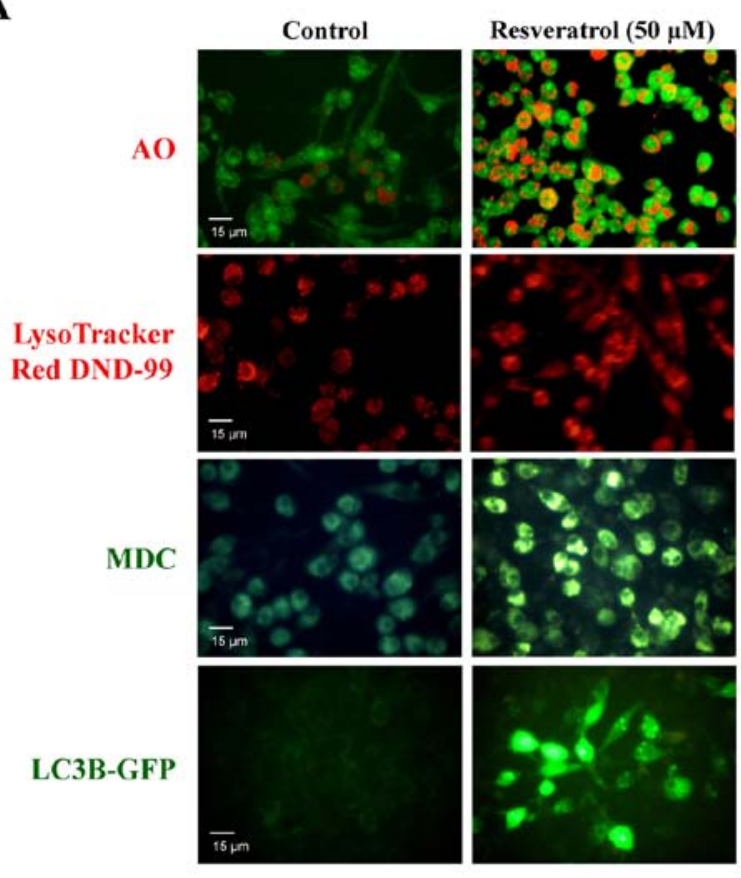

B

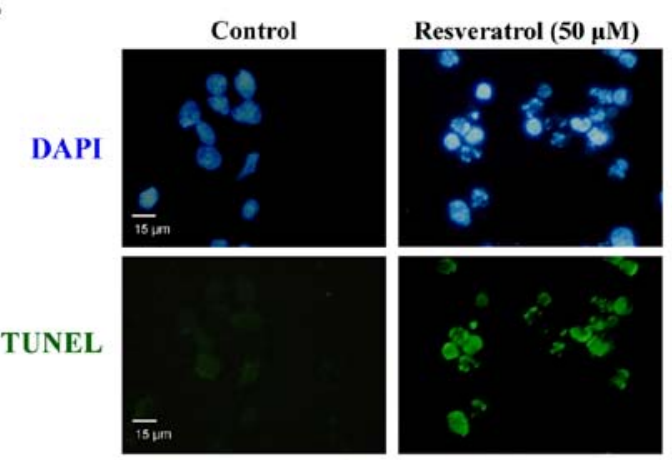

C

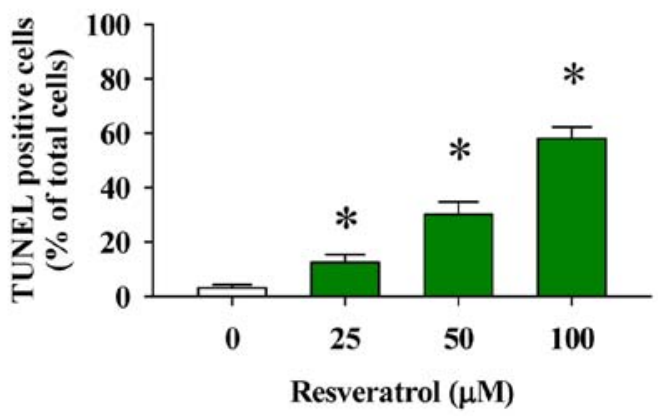

Figure 2. Effect of resveratrol on autophagic and apoptotic evidence of CAR cells. Cells with or without $50 \mu \mathrm{M}$ resveratrol challenge for $48 \mathrm{~h}$ were harvested. (A) Acridine orange (AO), LysoTracker Red DND-99, monodansylcadaverine (MDC) staining and LC3B-GFP kit were applied to detect acidic vesicular organelles (AVOs), autophagic vacuoles and LC3B expression, respectively, as described in Materials and methods. (B) DNA condensation and apoptotic DNA breaks were determined utilizing DAPI and TUNEL staining. Scale bar, $15 \mu \mathrm{m}$. (C) TUNEL positive cells was quantified. The data are expressed as the mean \pm SEM $(n=3)$. $P<0.05$ compared to untreated control.

cells was also explored. A more punctate pattern of LC3-GFP was found in cells after resveratrol treatment (Fig. 2A). In addition, the occurrence of DNA fragmentation and condensation by TUNEL/DAPI staining was detected prior to $50 \mu \mathrm{M}$ resveratrol exposure for $48 \mathrm{~h}$. Resveratrol markedly provoked apoptotic DNA breaks (Fig. 2B), and quantitative data of TUNEL positive cells was concentration-dependent (Fig. 2C). Therefore, resveratrol-induced cell death was triggered by autophagic and apoptotic machineries in CAR cells.

Resveratrol regulates the AMPK, Akt and autophagy-related protein levels in CAR cells. The protein signals of AMPK and Akt pathway were clarified in resveratrol-treated cells. Resveratrol at 25, 50 and $100 \mu \mathrm{M}$ for $24 \mathrm{~h}$ increased the protein levels of phosphorylated AMPK $\alpha$ on Thr172 and AMPK $\alpha$, but it decreased the phosphorylation of Akt on Ser473 and mTOR on Ser2448 (Fig. 3A). Our findings indicate that resveratrol induced autophagic cell death through modulation of AMPK and Akt signaling. The key protein levels of autophagy marker were also monitored. Resveratrol at 25,50 and $100 \mu \mathrm{M}$ for $48 \mathrm{~h}$ increased the protein levels of Atg5, Atg7, Atg12, Atg14, Atg16L1, Beclin-1, PI3K class III and LC3-II, but it decreased Rubicon protein level (Fig. 3B). These data imply that resveratrol induced autophagy through interfering with the PI3K class III/Beclin-1/Atgs-associated signals in CAR cells.

Resveratrol stimulates autophagy-modulated $m R N A$ levels in CAR cells. The autophagy-related gene was explored prior to resveratrol exposure for $24 \mathrm{~h}$. Resveratrol enhanced the gene expression of Atg5 (Fig. 4A), Atg12 (Fig. 4B), Beclin-1 (Fig. 4C) and LC3-II (Fig. 4D) in CAR cells. Thus, the key autophagic effect on gene levels resulted from resveratrol challenge in CAR cells.

3-MA and compound $c$ are against the autophagic effects on resveratrol-treated $C A R$ cells. Cells were pretreated with $10 \mathrm{mM}$ 3-MA (an inhibitor of PI3K class III) or $10 \mu \mathrm{M}$ compound $\mathrm{c}$ (an AMPK inhibitor) and then exposed to $50 \mu \mathrm{M}$ resveratrol for $48 \mathrm{~h}$. The protein level of LC3-II and cell viability were monitored. Our data demonstrated that 3-MA and compound $\mathrm{c}$ inhibited the formation of LC3-II (Fig. 5A and C) and enhanced cell viability after resveratrol treatment (Fig. 5B and D) in comparison to resveratrol-treated sample, suggesting that resveratrol-caused autophagy was mediated through regulating AMPK and PI3K class III signaling in CAR cells.

Resveratrol modulates caspase-3, caspase-9 and apoptoticrelated protein levels in CAR cells. The levels of caspase-3, caspase-9 and apoptotic-related protein molecules were investigated. Resveratrol at 25,50 and $100 \mu \mathrm{M}$ for $48 \mathrm{~h}$ increased the protein levels of caspase-3/-9 (Fig. 6A), cytochrome $c$, Apaf-1, AIF, Endo G, Bax and Bad (Fig. 6B), while it decreased the protein level of Bcl-2 and phosphorylation of Bad on Ser136. Our findings reveal that resveratrol induced apoptotic CAR cell death through a mitochondria-dependent pathway.

Resveratrol elicits the caspase-3/-9 activities and protective effect of pan-caspase inhibitor (Z-VAD-FMK) against resveratrol-induced caspase-dependent apoptosis on CAR cells. To 
A

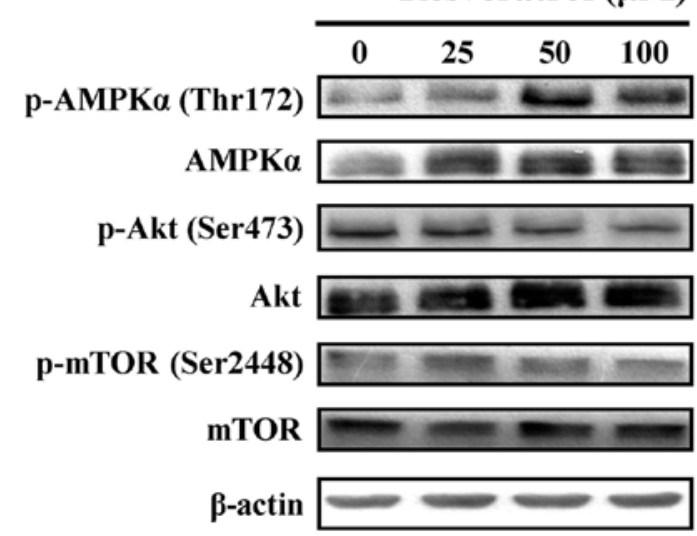

B

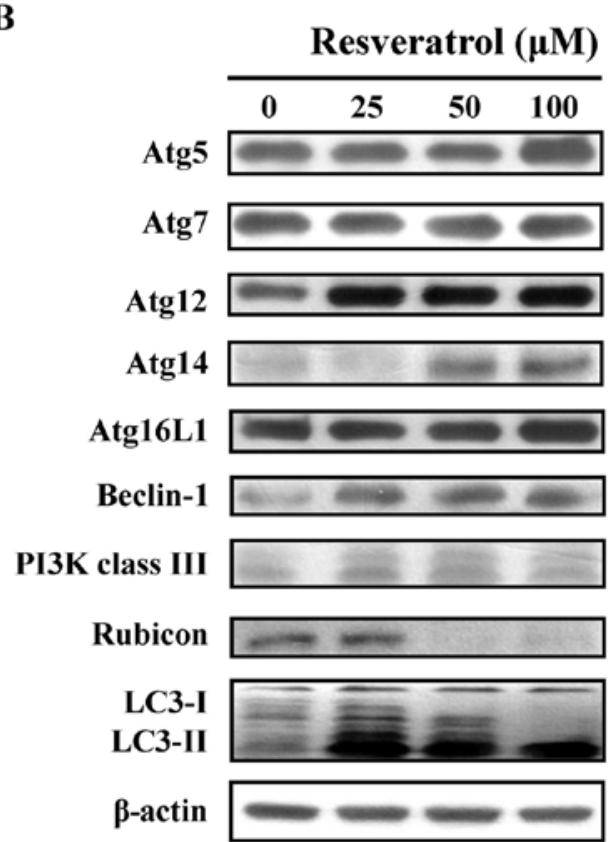

Figure 3. Effect of resveratrol on autophagy-regulated molecular signaling of CAR cells. The protein molecules from vehicle control or 25,50 and $100 \mu \mathrm{M}$ of resveratrol treatments in CAR cells for 24 or $48 \mathrm{~h}$ were verified using western blotting, as described in Materials and methods. Cell fractions were employed for the protein levels of (A) AMPK $\alpha$, phospho-AMPK $\alpha$ (Thr172), Akt, phospho-Akt (Ser473), mTOR and phospho-mTOR (Ser2448), as wells as (B) Atg5, Atg7, Atg12, Atg14, Atg16L1, Beclin-1, PI3K class III, Rubicon and LC3. $\beta$-actin is an internal control to ensure equal loading for each lane.
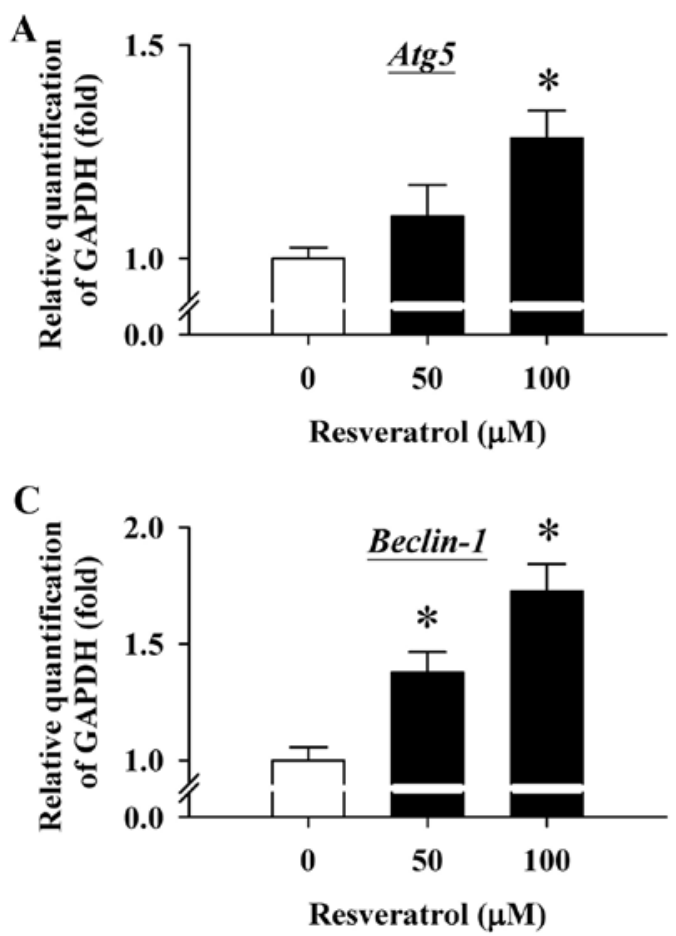
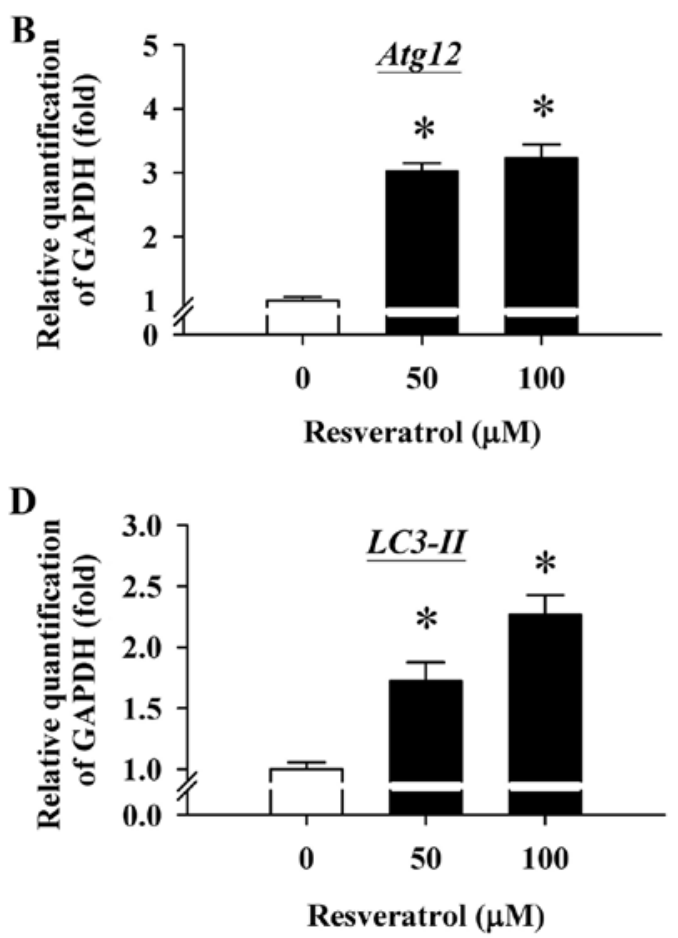

Figure 4. Effect of resveratrol on autophagy-related gene expression of CAR cells. Total RNA was extracted from CAR cells after 50 and $100 \mu \mathrm{M}$ of resveratrol treatments for $24 \mathrm{~h}$, and quantitative RT-PCR was carried out for the primers of (A) Atg5, (B) Atg12, (C) Beclin-1 and (D) LC3-II gene levels, respectively, as described in Materials and methods. The data are presented as the mean \pm SEM $(n=3)$. ${ }^{*} \mathrm{P}<0.05$ compared to untreated control.

further confirm the activity of caspase-3/-9 in resveratrol-treated cells, the caspase activities were detected. Cells were pretreated with $15 \mu \mathrm{M}$ Z-VAD-FMK and then exposed to $50 \mu \mathrm{M}$ resveratrol for $48 \mathrm{~h}$. The protein levels of cleaved caspase-9, cleaved caspase- 3 and cell viability were checked. Our data showed that resveratrol at 25,50 and $100 \mu \mathrm{M}$ for $48 \mathrm{~h}$ increased the activities of caspase-9 (Fig. 7A) and caspase-3 (Fig. 7B). Furthermore, Z-VAD-FMK inhibited the formation of cleaved caspase-9 and cleaved caspase-3 (Fig. 7C), as well as enhanced the viability of resveratrol-treated cells (Fig. 7D). Our results suggest that 
A

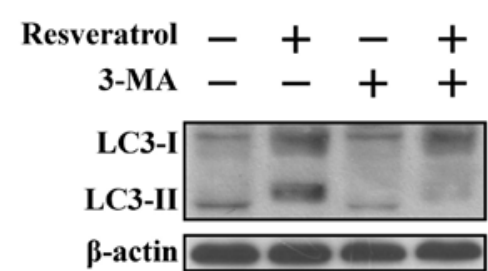

C

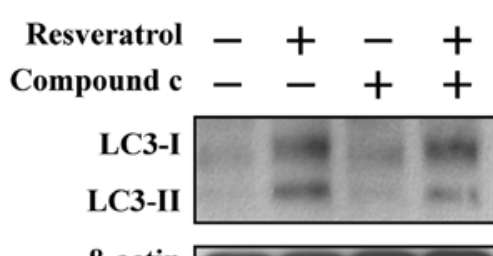

B

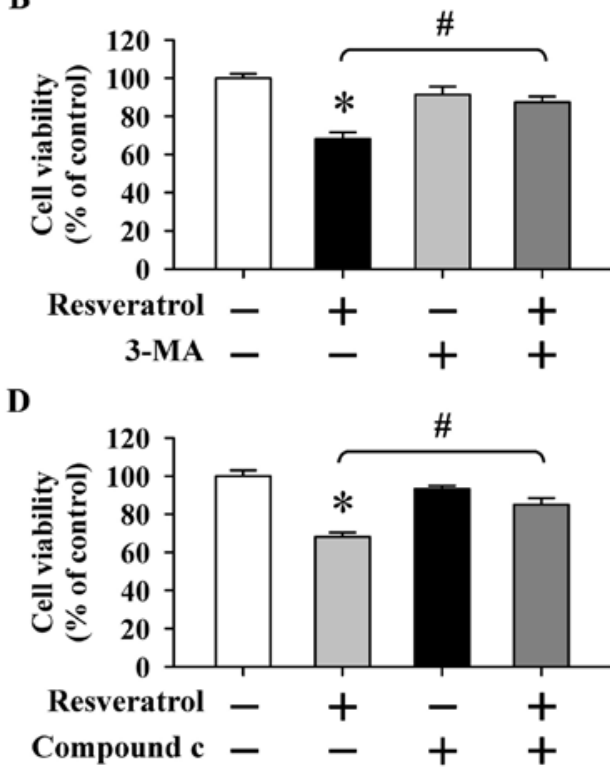

Figure 5. Effect of 3-MA and compound $\mathrm{c}$ on autophagic death in resveratrol-treated CAR cells. Cells were pre-incubated with or without $10 \mathrm{mM}$ 3-MA or $10 \mu \mathrm{M}$ compound c before exposure to $50 \mu \mathrm{M}$ resveratrol for $48 \mathrm{~h}$. (A and C) The LC3 protein level was assessed using western blotting. $\beta$-actin serves as an internal control. (B and D) MTT assay was applied to estimate cell viability of CAR cells. The data are given as the mean \pm SEM $(n=3)$. ${ }^{*} \mathrm{P}<0.05$ compared to untreated control; ${ }^{\#} \mathrm{P}<0.05$ compared to resveratrol-treated cells.

A

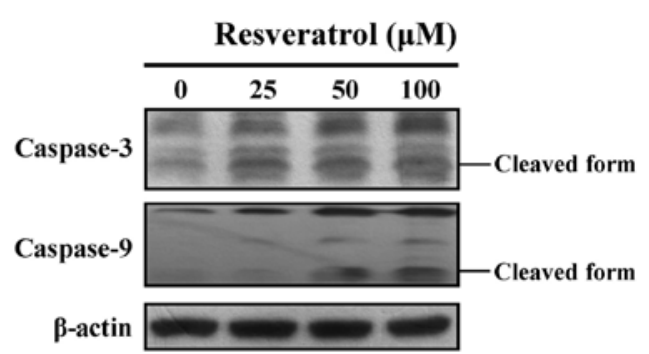

B

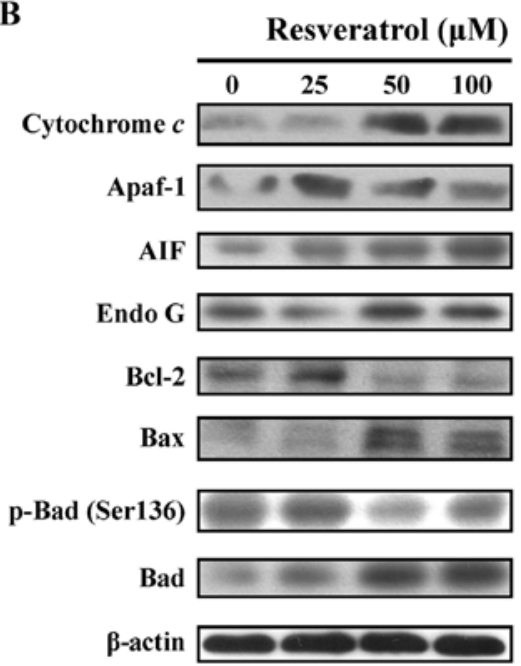

Figure 6. Effect of resveratrol on apoptotic signaling of CAR cells. Cells were treated with or without 25,50 and $100 \mu \mathrm{M}$ resveratrol for $48 \mathrm{~h}$, and cell lysates were collected and blotted using the specific antibodies, including (A) caspase-3/-9; (B) cytochrome $c$, Apaf-1, AIF, Endo G, Bcl-2, Bax, p-Bad (Ser136) and Bad by immunoblot analysis, as described in Materials and methods. Each lane of protein signaling is normalized to $\beta$-actin.

the intrinsic mechanism was required for resveratrol-induced apoptosis in CAR cells.

\section{Discussion}

Resveratrol has been shown to have a variety of biological actions and many health benefits $(18,19)$. It is well documented that resveratrol is an effective anti-oxidant with anti-neoplastic potential for cell cycle arrest and apoptosis (16,33-36). Furthermore, resveratrol has been shown to induce autophagic cell death in ovarian $(33,34)$ and colon $(35,36)$ cancer cells. Many studies showed that resveratrol-induced tumor cell death may be mediated through autophagy and apoptosis $(34,37,38)$. Our study is first to demonstrate that resveratrol triggered cell autophagy and apoptosis in cisplatin-resistant human oral cancer CAR cells.

In the present study, our results revealed that the resveratrol induced the formation of autophagic vesicle, AVOs and LC3B occurrence (Fig. 2A) in CAR cells. LC3 is a hallmark of autophagic membrane to monitor early autophagosome formation (37). It is important to note that CAR cell morphology prior to resveratrol treatment showed strictly apoptotic action, and intensive accumulation of autophagic vacuoles was also detected. It started before pronounced hallmarks of apoptosis appeared, suggesting that the autophagic pathway switched to the apoptotic machinery (Fig. 2). Noteworthy, resveratrol was reported to induce both autophagy and apoptosis in human bladder and breast cancer cells $(39,40)$. Moreover, resveratrol also induced autophagy followed by apoptosis in human colorectal DLD1 cancer cells (41). Thus, our study is in agreement with previous findings (39-41).

In addition, morphology of resveratrol-treated cells could be affected by both apoptosis and presumably defective autophagy. Resveratrol also simultaneously enhanced the protein level of autophagy-associated proteins (Atg complexes, Beclin-1, PI3K class III and LC3-II) (Fig. 3B), and mRNA expression of autophagic genes Atg5, Atg12, Beclin-1 and LC3-II (Fig. 4) in CAR cells. 3-MA (an inhibitor of PI3K class III) has been shown to 
A

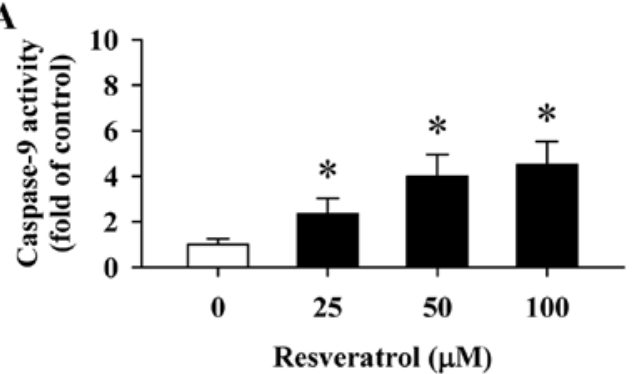

C

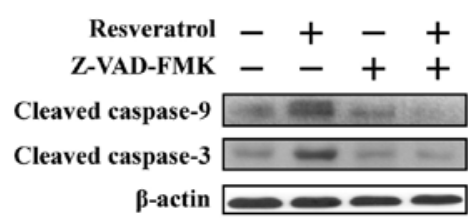

B

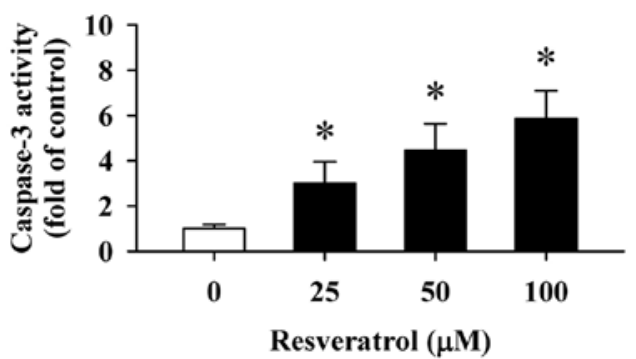

D

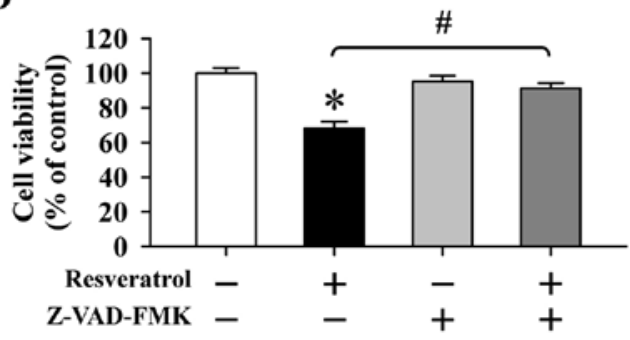

Figure 7. Effect of Z-VAD-FMK on apoptotic death and caspase activities of resveratrol-treated CAR cells. Cells were pretreated in the presence or absence of $15 \mu \mathrm{M}$ Z-VAD-FMK (a pan-caspase inhibitor) and then exposed to $50 \mu \mathrm{M}$ resveratrol for $48 \mathrm{~h}$. The activities of (A) caspase- 9 and (B) caspase-3 were estimated, as described in Materials and methods. (C) The protein levels of caspase-3/-9 were determined by western blotting. (D) Cell viability was assessed by MTT assay. The data are shown as the mean $\pm \operatorname{SEM}(n=3) .{ }^{*} \mathrm{P}<0.05$ compared to untreated control; ${ }^{*} \mathrm{P}<0.05$ compared to resveratrol-treated cells.

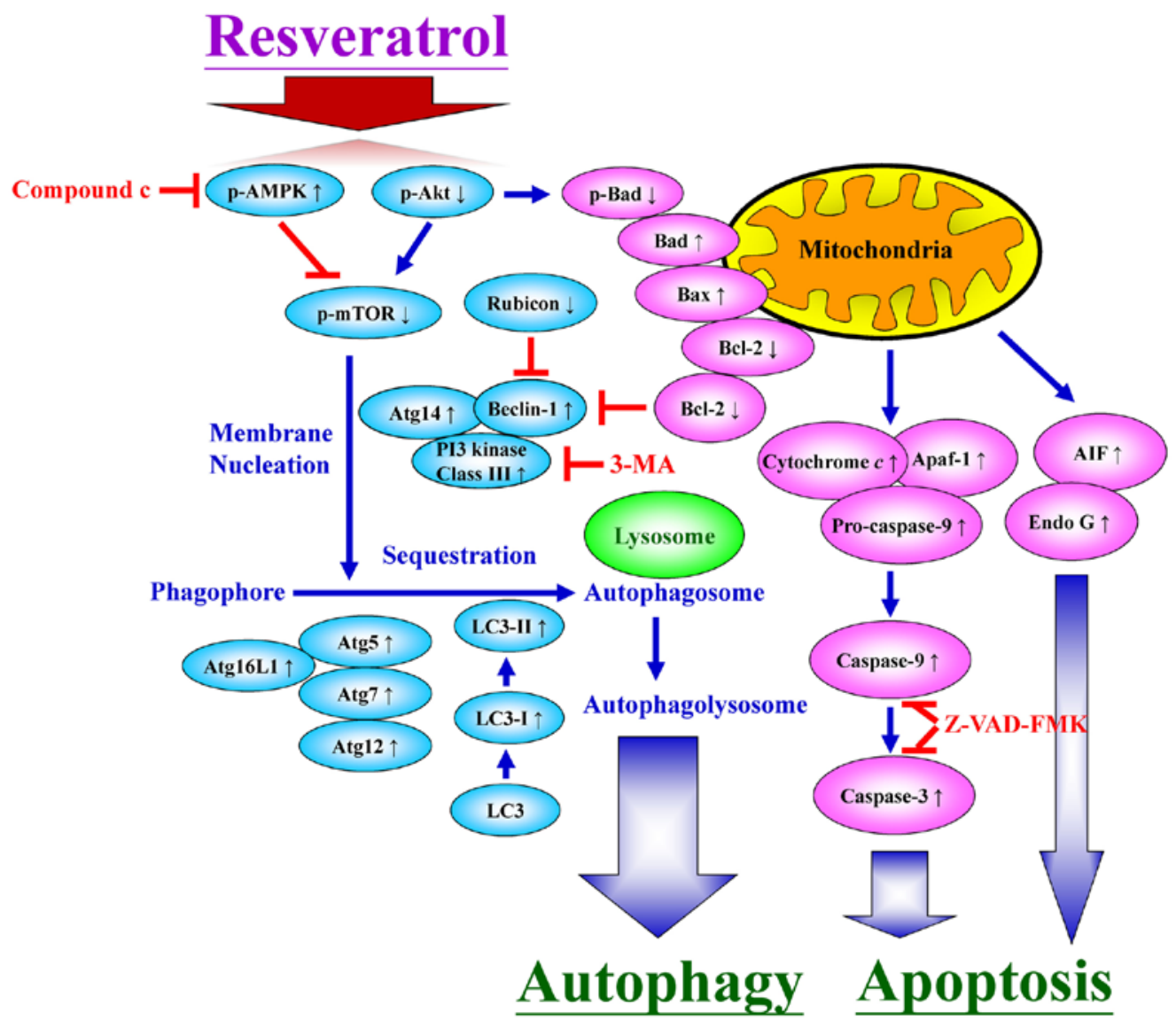

Figure 8. Schematic diagram of proposed molecular mechanism of resveratrol-provoked autophagy and apoptosis in cisplatin-resistant human oral cancer CAR cells.

potently inhibit autophagy-dependent protein degradation and to suppress the formation of autophagosomes (42). Importantly, our results showed that 3-MA inhibited the autophagic vesicle formation and LC3-II protein expression induced by resveratrol (Fig. 5A). Autophagy (programmed cell death type II) plays a double role in carcinogenesis, while apoptosis is well 
known as programmed cell death type I $(11,12)$. Cells undergoing autophagic cell death display distinct morphological features $(1,43)$. In contrast, autophagy is early degradation of organelles whereas preservation of nucleus until late stages.

Moreover, autophagy serves as a key mechanism of cell survival and is able to represent an adaptive mechanism to survive drug-induced cellular stress in tumor cells $(11,12)$. It has been reported that autophagy precedes apoptosis in resveratrol-treated cervical cancer HeLa and Cx cells (44), suggesting that resveratrol might lead to apoptotic cell death through autophagy. Resveratrol induced autophagy in HT-29 and COLO 201 cells, and the magnitude of resveratrol-induced apoptosis and autophagy was different upon colon cancer cell lines (45). In the present study, CAR cells were used as an experimental model on cellular mechanisms. Therefore, we demonstrated that resveratrol may act as a modulator of autophagy in CAR cells.

Resveratrol and other polyphenols exhibit antioxidant properties (16-18). The formation of autophagic vacuoles in resveratrol-treated CAR cells was blocked by 3-MA to inhibit the sequestration step of macroautophagy, i.e. autophagosome formation (data not shown). An increased level of LC3-II was caused by resveratrol treatment (Fig. 3B) in CAR cells. This indicated that the conversion of LC3-I to LC3-II, typically observed after induction of autophagy, reflecting increased number of autophagosomes due to an elevated activity of autophagic process $(37,41)$. The elevated amount of LC3-I in CAR cells after a 24-h treatment suggests that its processed LC3-II form is still required. Furthermore, accumulation of autophagic vacuoles in resveratrol-treated cells was observed by detecting LC3-II positive CAR cells (Fig. 2A).

In this study, our data demonstrated that resveratrol induced autophagic cell death through inhibition of Akt and enhancement of AMPK signaling in CAR cells (Fig. 3A). The abnormal activation of $\mathrm{Akt} / \mathrm{mTOR}$ signaling cascade that stimulates tumor cell growth, proliferation, survival and resistance to drug-induced apoptosis (46). Currently, autophagy regulation is mediated through PI3K/Akt/mTOR signal pathway $(11,12)$. AMPK and Akt serine/threonine kinase [protein kinase B (PKB)] are vital to be involved in the induction of cell autophagy and apoptosis (47). Regulation of the Akt signaling is essential for developing therapeutic inhibitors for tumor cells (13). Zhao et al (48) showed that Akt inhibitor (MK-2206) induces autophagy in human nasopharyngeal cancer NPC cells in vitro. It is also reported that the polyphenolic compound plumbagin triggers autophagic and apoptotic cell death through suppressing Akt pathway in human non-small cell lung cancer cells (49). AMPK activation promotes autophagy by directly activating Ulk1 through direct phosphorylation of Ser317 and Ser777 because a key energy sensor regulates cellular metabolism to maintain energy homeostasis $(50,51)$.

We suggest herein that AMPK activation by resveratrol directly phosphorylates Ulk1 on Ser317 to mediate cell autophagy, which also contributed to CAR cell apoptosis. As previously mentioned, multiple antitumor agents, including vincristine, taxol and doxorubicin, activate AMPK to promote cancer cell death (52). Din et al (53) showed that aspirin elicits autophagy cell death through activation of AMPK and inhibition of mTOR activation in colorectal cancer cells in vivo and in vitro. Furthermore, Liu et al (54) also demonstrated that AMPK activator AA005 induces mTORC1 inhibition and autophagy cell death in colon cancer cells. These results are similar to our findings presented here. Hence, resveratrol exerts the effects of autophagy and apoptosis by altering the activation of Akt and AMPK signaling.

Apoptosis is associated with the disruption of mitochondrial membrane potential $(\Delta \Psi \mathrm{m})$, and the mitochondrion integrate cell death signals and autophagy activation $(6,55)$. Apoptosis and autophagic cell death are interconnected through mitochondrial permeability transition (16), and the activation of caspase- 3 and increased release of cytochrome $c$ contribute to resveratrol-triggered apoptosis. Our data indicated a rapid collapse of $\Delta \Psi \mathrm{m}$ after resveratrol challenge at an early stage (data not shown). The loss of mitochondrial membrane polarization seems to be an intracellular signal that may be responsible for the induction of autophagy and apoptosis (10). Resveratrol led to phosphatidylserine externalization as well as disruption of the mitochondrial membrane potential, activation of caspase-9/-3 and DNA degradation (Figs. 2B, 6A and 7). Z-VAD-FMK (a pan-caspase inhibitor) has been shown to potently inhibit apoptosis-dependent caspase activity (32). Resveratrol-induced cell death was effectively inhibited by Z-VAD-FMK, indicating the involvement of the mitochondrial apoptotic pathway (Fig. 8). These findings provide evidence for induction of apoptosis in CAR cells after resveratrol challenge.

In summary, our findings suggest that treatment of CAR cells with resveratrol caused reduction rather than enhancement of autophagic degradation, which in turn leads to cell death. Autophagy is initiated in resveratrol-treated CAR cells, but removal of autophagic vacuoles is probably inefficient. Further studies are required to fully explain this phenomenon. Our studies provide new insight into the molecular mechanism of action of resveratrol and proposes its potential therapeutic use. Understanding the underlying pathways affected by resveratrol in CAR cells may help to identify new targets for effective cancer therapy or predict potential adverse drug effects.

\section{Acknowledgements}

This study was supported by the grant from China Medical University Hospital, Taichung, Taiwan (DMR-105-096).

\section{References}

1. Inoue $\mathrm{H}$ and Tani K: Multimodal immunogenic cancer cell death as a consequence of anticancer cytotoxic treatments. Cell Death Differ 21: 39-49, 2014.

2. Plati J, Bucur O and Khosravi-Far R: Apoptotic cell signaling in cancer progression and therapy. Integr Biol 3: 279-296, 2011.

3. Shen HM and Codogno P: Autophagic cell death: Loch Ness monster or endangered species? Autophagy 7: 457-465, 2011.

4. Shimizu S, Yoshida T, Tsujioka M and Arakawa S: Autophagic cell death and cancer. Int J Mol Sci 15: 3145-3153, 2014.

5. Pereira DL, Dos Santos Ferreira AC, de Faria GP and Kwee JK: Autophagy interplays with apoptosis and cell cycle regulation in the growth inhibiting effect of Trisenox in HEP-2, a laryngeal squamous cancer. Pathol Oncol Res 21: 103-111, 2015.

6. Huang WW, Chiu YJ, Fan MJ, Lu HF, Yeh HF, Li KH, Chen PY, Chung JG and Yang JS: Kaempferol induced apoptosis via endoplasmic reticulum stress and mitochondria-dependent pathway in human osteosarcoma U-2 OS cells. Mol Nutr Food Res 54: $1585-1595,2010$ 
7. Xiong S, Mu T, Wang G and Jiang X: Mitochondria-mediated apoptosis in mammals. Protein Cell 5: 737-749, 2014.

8. Yeh CC, Ko HH, Hsieh YP, Wu KJ, Kuo MY and Deng YT: Phenethyl isothiocyanate enhances TRAIL-induced apoptosis in oral cancer cells and xenografts. Clin Oral Investig 20 2343-2352, 2016.

9. Ho TF and Chang CC: A promising 'TRAIL' of tanshinones for cancer therapy. Biomedicine (Taipei) 5: 23, 2015.

10. Kong B, Tsuyoshi H, Orisaka M, Shieh DB, Yoshida Y and Tsang BK: Mitochondrial dynamics regulating chemoresistance in gynecological cancers. Ann NY Acad Sci 1350: 1-16, 2015.

11. Fu Y, Chang H, Peng X, Bai Q, Yi L, Zhou Y, Zhu J and Mi M Resveratrol inhibits breast cancer stem-like cells and induces autophagy via suppressing $\mathrm{Wnt} / \beta$-catenin signaling pathway. PLoS One 9: e102535, 2014.

12. Baehrecke EH: Autophagy: Dual roles in life and death? Nat Rev Mol Cell Biol 6: 505-510, 2005.

13. Hsieh MT, Chen HP, Lu CC, Chiang JH, Wu TS, Kuo DH, Huang LJ, Kuo SC and Yang JS: The novel pterostilbene derivative ANK-199 induces autophagic cell death through regulating PI3 kinase class III/beclin 1/Atg-related proteins in cisplatin-resistant CAR human oral cancer cells. Int J Oncol 45 782-794, 2014

14. Adhauliya N, Kalappanavar AN, Ali IM and Annigeri RG: Autophagy: A boon or bane in oral cancer. Oral Oncol 61: 120-126, 2016

15. Sui X, Chen R, Wang Z, Huang Z, Kong N, Zhang M, Han W, Lou F, Yang J, Zhang Q, et al: Autophagy and chemotherapy resistance: A promising therapeutic target for cancer treatment. Cell Death Dis 4: e838,2013.

16. Varoni EM, Lo Faro AF, Sharifi-Rad J and Iriti M: Anticancer molecular mechanisms of resveratrol. Front Nutr 3: 8, 2016.

17. de Oliveira MR, Nabavi SF, Manayi A, Daglia M, Hajheydari Z and Nabavi SM: Resveratrol and the mitochondria: From triggering the intrinsic apoptotic pathway to inducing mitochondria biogenesis, a mechanistic view. Biochim Biophys Acta 1860 727-745, 2016.

18. Marques FZ, Markus MA and Morris BJ: Resveratrol: Cellular actions of a potent natural chemical that confers a diversity of health benefits. Int J Biochem Cell Biol 41: 2125-2128, 2009.

19. Gehm BD, McAndrews JM, Chien PY and Jameson JL: Resveratrol, a polyphenolic compound found in grapes and wine, is an agonist for the estrogen receptor. Proc Natl Acad Sci USA 94: 14138-14143, 1997.

20. Dandawate PR, Subramaniam D, Jensen RA and Anant S: Targeting cancer stem cells and signaling pathways by phytochemicals: Novel approach for breast cancer therapy. Semin Cancer Biol 40-41: 192-208, 2016.

21. Jang M, Cai L, Udeani GO, Slowing KV, Thomas CF, Beecher CW, Fong HH, Farnsworth NR, Kinghorn AD, Mehta RG, et al: Cancer chemopreventive activity of resveratrol, a natural product derived from grapes. Science 275: 218-220, 1997.

22. Subramanian L, Youssef S, Bhattacharya S, Kenealey J, Polans AS and van Ginkel PR: Resveratrol: challenges in translation to the clinic - a critical discussion. Clin Cancer Res 16 : 5942-5948, 2010

23. Soleas GJ, Diamandis EP and Goldberg DM: Resveratrol: A molecule whose time has come? And gone? Clin Biochem 30 91-113, 1997.

24. Gosepath EM, Eckstein N, Hamacher A, Servan K von Jonquieres G, Lage H, Györffy B, Royer HD and Kassack MU: Acquired cisplatin resistance in the head-neck cancer cell line Cal27 is associated with decreased DKK1 expression and can partially be reversed by overexpression of DKK1. Int J Cancer 123: 2013-2019, 2008.

25. Chang PY, Peng SF, Lee CY, Lu CC, Tsai SC, Shieh TM, Wu TS, Tu MG, Chen MY and Yang JS: Curcumin-loaded nanoparticles induce apoptotic cell death through regulation of the function of MDR1 and reactive oxygen species in cisplatin-resistant CAR human oral cancer cells. Int J Oncol 43: 1141-1150, 2013.

26. Shih YH, Lin DJ, Chang KW, Hsia SM, Ko SY, Lee SY, Hsue SS, Wang TH, Chen YL and Shieh TM: Evaluation physical characteristics and comparison antimicrobial and anti-inflammation potentials of dental root canal sealers containing hinokitiol in vitro. PLoS One 9: e94941, 2014.

27. Lu CC, Huang BR, Liao PJ and Yen GC: Ursolic acid triggers nonprogrammed death (necrosis) in human glioblastoma multiforme DBTRG-05MG cells through MPT pore opening and ATP decline. Mol Nutr Food Res 58: 2146-2156, 2014.
28. Ma YS, Weng SW, Lin MW, Lu CC, Chiang JH, Yang JS, Lai KC, Lin JP, Tang NY, Lin JG, et al: Antitumor effects of emodin on LS1034 human colon cancer cells in vitro and in vivo: Roles of apoptotic cell death and LS1034 tumor xenografts model. Food Chem Toxicol 50: 1271-1278, 2012

29. Chiang JH, Yang JS, Lu CC, Hour MJ, Chang SJ, Lee TH and Chung JG: Newly synthesized quinazolinone HMJ-38 suppresses angiogenetic responses and triggers human umbilical vein endothelial cell apoptosis through p53-modulated Fas/death receptor signaling. Toxicol Appl Pharmacol 269: 150-162, 2013.

30. Yuan CH, Horng CT, Lee CF, Chiang NN, Tsai FJ, Lu CC, Chiang JH, Hsu YM, Yang JS and Chen FA: Epigallocatechin gallate sensitizes cisplatin-resistant oral cancer CAR cell apoptosis and autophagy through stimulating AKT/STAT3 pathway and suppressing multidrug resistance 1 signaling. Environ Toxicol: May 20, 2016 (Epub ahead of print).

31. Lai KC, Huang AC, Hsu SC, Kuo CL, Yang JS, Wu SH and Chung JG: Benzyl isothiocyanate (BITC) inhibits migration and invasion of human colon cancer HT29 cells by inhibiting matrix metalloproteinase-2/-9 and urokinase plasminogen (uPA) through PKC and MAPK signaling pathway. J Agric Food Chem 58: 2935-2942, 2010

32. Lu CC, Yang JS, Chiang JH, Hour MJ, Lin KL, Lee TH and Chung JG: Cell death caused by quinazolinone HMJ-38 challenge in oral carcinoma CAL 27 cells: Dissections of endoplasmic reticulum stress, mitochondrial dysfunction and tumor xenografts. Biochim Biophys Acta 1840: 2310-2320, 2014.

33. Gwak H, Kim S, Dhanasekaran DN and Song YS: Resveratrol triggers ER stress-mediated apoptosis by disrupting N-linked glycosylation of proteins in ovarian cancer cells. Cancer Lett 371: 347-353, 2016.

34. Lang F, Qin Z, Li F, Zhang H, Fang Z and Hao E: Apoptotic cell death induced by resveratrol is partially mediated by the autophagy pathway in human ovarian cancer cells. PLoS One 10: e0129196, 2015.

35. Yuan SX, Wang DX, Wu QX, Ren CM, Li Y, Chen QZ, Zeng YH, Shao Y, Yang JQ, Bai Y, et al: BMP9/p38 MAPK is essential for the antiproliferative effect of resveratrol on human colon cancer. Oncol Rep 35: 939-947, 2016.

36. Crek C, Mutlu Altundag E, Karademir B, Kocturk S, Kartal Ozer N and Taga Y: The effect of resveratrol on signal transduction pathways and the role of pro-apoptotic Bax protein on apoptosis in HCT-116 colon carcinoma cell lines. Free Radic Biol Med 75 (Suppl 1): S27, 2014

37. Zhu Y, He W, Gao X, Li B, Mei C, Xu R and Chen H: Resveratrol overcomes gefitinib resistance by increasing the intracellular gefitinib concentration and triggering apoptosis, autophagy and senescence in PC9/G NSCLC cells. Sci Rep 5: 17730, 2015.

38. Selvaraj S, Sun Y, Sukumaran P and Singh BB: Resveratrol activates autophagic cell death in prostate cancer cells via downregulation of STIM1 and the mTOR pathway. Mol Carcinog 55: 818-831, 2016.

39. Alayev A, Berger SM, Kramer MY, Schwartz NS and Holz MK: The combination of rapamycin and resveratrol blocks autophagy and induces apoptosis in breast cancer cells. J Cell Biochem 116: 450-457, 2015

40. Lin X, Wu G, Huo WQ, Zhang Y and Jin FS: Resveratrol induces apoptosis associated with mitochondrial dysfunction in bladder carcinoma cells. Int J Urol 19: 757-764, 2012.

41. Trincheri NF, Follo C, Nicotra G, Peracchio C, Castino R and Isidoro C: Resveratrol-induced apoptosis depends on the lipid kinase activity of Vps34 and on the formation of autophagolysosomes. Carcinogenesis 29: 381-389, 2008.

42. Button RW, Vincent JH, Strang CJ and Luo S: Dual PI-3 kinase/mTOR inhibition impairs autophagy flux and induces cell death independent of apoptosis and necroptosis. Oncotarget 7: 5157-5175, 2016.

43. Elmore S: Apoptosis: A review of programmed cell death Toxicol Pathol 35: 495-516, 2007.

44. García-Zepeda SP, García-Villa E, Díaz-Chávez J, Hernández-Pando R and Gariglio P: Resveratrol induces cell death in cervical cancer cells through apoptosis and autophagy. Eur J Cancer Prev 22: 577-584, 2013.

45. Miki H, Uehara N, Kimura A, Sasaki T, Yuri T, Yoshizawa K and Tsubura A: Resveratrol induces apoptosis via ROS-triggered autophagy in human colon cancer cells. Int J Oncol 40: 1020-1028, 2012

46. Mancias JD and Kimmelman AC: Targeting autophagy addiction in cancer. Oncotarget 2: 1302-1306, 2011.

47. He C and Klionsky DJ: Regulation mechanisms and signaling pathways of autophagy. Annu Rev Genet 43: 67-93, 2009. 
48. Zhao YY, Tian Y, Zhang J, Xu F, Yang YP, Huang Y, Zhao HY, Zhang JW, Xue C, Lam MH, et al: Effects of an oral allosteric AKT inhibitor (MK-2206) on human nasopharyngeal cancer in vitro and in vivo. Drug Des Devel Ther 8: 1827-1837, 2014.

49. Li YC, He SM, He ZX, Li M, Yang Y, Pang JX, Zhang X, Chow K, Zhou Q, Duan W, et al: Plumbagin induces apoptotic and autophagic cell death through inhibition of the PI3K/Akt/mTOR pathway in human non-small cell lung cancer cells. Cancer Lett 344: 239-259, 2014.

50. Kim J, Kundu M, Viollet B and Guan KL: AMPK and mTOR regulate autophagy through direct phosphorylation of Ulk1. Nat Cell Biol 13: 132-141, 2011.

51. Zhu LQ, Zhen YF, Zhang Y, Guo ZX, Dai J and Wang XD: Salinomycin activates AMP-activated protein kinase-dependent autophagy in cultured osteoblastoma cells: A negative regulator against cell apoptosis. PLoS One 8: e84175, 2013.

52. Jiang H, Liu W, Zhan SK, Pan YX, Bian LG, Sun B, Sun QF and Pan SJ: GSK621 targets glioma cells via activating AMP-activated protein kinase signalings. PLoS One 11: e0161017, 2016.
53. Din FV, Valanciute A, Houde VP, Zibrova D, Green KA, Sakamoto K, Alessi DR and Dunlop MG: Aspirin inhibits mTOR signaling, activates AMP-activated protein kinase, and induces autophagy in colorectal cancer cells. Gastroenterology 142: 1504-1515.e3, 2012.

54. Liu YQ, Cheng X, Guo LX, Mao C, Chen YJ, Liu HX, Xiao QC, Jiang S, Yao ZJ and Zhou GB: Identification of an annonaceous acetogenin mimetic, AA005, as an AMPK activator and autophagy inducer in colon cancer cells. PLoS One 7: e47049, 2012.

55. Yang JS, Chen GW, Hsia TC, Ho HC, Ho CC, Lin MW, Lin SS, Yeh RD, Ip SW, Lu HF, et al: Diallyl disulfide induces apoptosis in human colon cancer cell line (COLO 205) through the induction of reactive oxygen species, endoplasmic reticulum stress, caspases casade and mitochondrial-dependent pathways. Food Chem Toxicol 47: 171-179, 2009. 